\title{
Copeptin is independently associated with vascular calcification in chronic kidney disease stage 5
}

\author{
Edyta Golembiewska ${ }^{1,2^{*}}$, Abdul Rashid Qureshi', Lu Dai ${ }^{1}$, Bengt Lindholm¹, Olof Heimbürger ${ }^{1}$, Magnus Söderberg $^{3}$,
} Torkel B. Brismarr , Jonaz Ripsweden ${ }^{4}$, Peter Barany ${ }^{1}$, Richard J. Johnson ${ }^{5}$ and Peter Stenvinkel ${ }^{1}$

\begin{abstract}
Background: Vascular calcification (VC) is an independent predictor of cardiovascular disease (CVD) present in 30$70 \%$ of patients with chronic kidney disease (CKD). Copeptin is a sensitive surrogate marker of arginine vasopressin (AVP), which is involved in many pathophysiologic processes in CKD. The aim of the present study was to explore the association of copeptin with VC in CKD stage 5.

Methods: Copeptin was investigated in conjunction with living donor kidney transplantation in 149 clinically stable CKD stage 5 patients (CKD5), including 53 non-dialyzed (CKD5-ND) and 96 dialysis patients treated by peritoneal dialysis (PD) $(n=43)$ or hemodialysis (HD) $(n=53)$. We analyzed the association of copeptin with presence and extent of VC ascertained both histologically in biopsies from the inferior epigastric artery $(n=137)$ and by coronary artery calcification (CAC) score measured by computed tomography.

Results: Patients with higher copeptin were older, had higher systolic blood pressure, higher prevalence of CVD and their preceding time on chronic dialysis was longer. In Spearman's rank correlations (Rho), copeptin concentrations were significantly associated with CAC score (Rho $=0.27 ; p=0.003)$ and presence of medial VC $($ Rho $=0.21 ; p=0.016$ ). Multivariate logistic regression analysis showed that 1-SD higher age, male gender, diabetes and 1-SD higher copeptin were significantly associated with the presence of moderate-extensive VC.

Conclusions: High circulating levels of copeptin in CKD5 patients are independently associated with the degree of medial calcification ascertained by histology of arterial biopsies. Thus, plasma copeptin may serve as a marker of the uremic calcification process.
\end{abstract}

Keywords: Copeptin, Chronic kidney disease, End-stage renal disease, Vascular calcification

\section{Background}

Chronic kidney disease (CKD) is associated with markedly increased morbidity and mortality which is mainly a result of premature cardiovascular disease (CVD) [1-3]. The causes for the high prevalence of CVD are still not fully elucidated and cannot be explained only by traditional Framingham risk factors, such as age, hypertension, diabetes

\footnotetext{
* Correspondence: irys@pum.edu.pl

'Division of Renal Medicine and Baxter Novum, Department of Clinical Science, Intervention and Technology, Karolinska Institutet, Campus Flemingsberg, Stockholm, Sweden

${ }^{2}$ Department of Nephrology, Transplantology and Internal Medicine, Pomeranian Medical University, Al. Powstancow Wlkp. 72, 70-111 Szczecin, Poland

Full list of author information is available at the end of the article
}

mellitus, dyslipidemia and smoking. In addition, CKDspecific risk factors, e.g. inflammation, prooxidantantioxidant imbalance, protein-energy wasting, disorders in mineral metabolism and gut dysbiosis, also play a role [2, 4].

Medial vascular calcification (VC), i.e. calcific arteriosclerosis, an independent predictor of CVD, is reported in $30-70 \%$ of adult CKD patients, and $\approx 15 \%$ of pediatric CKD patients [5, 6]. VC develops in the intima together with atherosclerosis, which is defined as an increase in the deposition of plaques, and in the media together with arteriosclerosis, which is associated with vascular stiffening resulting from the loss of elasticity of muscle layer of the arterial wall. The presence of VC results in arterial dysfunction, such as NO-dependent endothelial

(C) The Author(s). 2020 Open Access This article is distributed under the terms of the Creative Commons Attribution 4.0 International License (http://creativecommons.org/licenses/by/4.0/), which permits unrestricted use, distribution, and 
dysfunction [7]. The extent of coronary artery calcification (CAC) examined by cardiac computed tomography (CT) is a useful tool in assessing extent of intima and medial VC $[8,9]$. Medial calcification is predominantly related to abnormal metabolism in CKD and represents one of the uremic features entitled CKD - mineral and bone disorder (CKD-MBD) [10]. Although several biomarkers, such as calcium, phosphate, alkaline phosphatase, parathyroid hormone, fibroblast growth factor-23 (FGF-23), Klotho, vitamin $\mathrm{D}_{3}$, osteoprotegerin (OPG) and sclerostin, play important roles in pathological pathways of CKD-BMD [11-13], the exact pathogenic mechanisms remain unclear and additional factors are likely to be involved.

Recently, there is increased attention to the role of the arginine vasopressin (AVP) system, particularly AVP and copeptin, in the pathophysiological mechanisms of CKD. AVP, known also as antidiuretic hormone, is produced in the hypothalamus in the form of a precursor, preproAVP, and released from the posterior pituitary gland in response to different osmotic and non-osmotic forces. The principal role of AVP is the control of fluid balance by promoting water preservation by the kidney, but, in addition, AVP is involved in the mediation of arteriolar vasoconstriction and contributes to cardiovascular stability [14]. Copeptin, a 39-amino acid glycopeptide with a leucine-rich core segment and molecular mass of $5 \mathrm{kDa}$ is derived from the C-terminal portion of the preprovasopressin and is co-released with AVP in equimolar amounts. Copeptin is thought to be a reliable, sensitive and easy to measure surrogate marker of AVP [15]. This observation has prompted extensive research regarding copeptin - as a surrogate measure of AVP - in numerous clinical situations. High circulating copeptin is associated with decline in glomerular filtration rate and a greater risk of new-onset CKD [16, 17]. Moreover, high copeptin has been linked with increased risk of myocardial infarction, heart failure, hypertension, ischemic stroke, diabetes mellitus and metabolic syndrome $[14,18]$. In patients with type 1 diabetes, elevated copeptin levels were strongly related to diabetic CKD and coronary atherosclerosis [19].

The aim of the present study was to examine the association of copeptin with the presence and extent of CAC (by $\mathrm{CT}$ ) and medial $\mathrm{VC}$ ascertained in biopsies from the inferior epigastric artery in CKD5 patients at the time of living donor kidney transplantation (LD-Rtx).

\section{Methods}

\section{Patients and study design}

Circulating concentrations of copeptin were measured in clinically stable CKD 5 patients $(n=149)$ comprising non-dialyzed patients (CKD5-ND; $n=53$ ), and dialysis patients $(n=96)$ treated by peritoneal dialysis (PD; $n=$
43) or hemodialysis (HD; $n=53$ ) recruited from an ongoing study on vascular changes before undergoing LDRtx [13]. Their age ranged between 19 and 75 years, 101 (68\%) were men, 16 (11\%) had diabetes mellitus and 23 (15\%) had previously been diagnosed with cerebrovascular, cardiovascular, and/or peripheral vascular disease (grouped as CVD).

The characteristics of patients, according to tertiles of copeptin, are shown in Table 1. Patients were recruited from March 2009 to February 2017. Exclusion criterion was unwillingness to participate. Informed consent to participate in the study - including consent to biopsy of inferior epigastric artery during transplantation procedure - was obtained from each patient. The Ethics Committee of the Karolinska Institutet (EPN) approved study protocols. The studies were conducted in adherence to the Declaration of Helsinki.

\section{CKD5-ND patients}

The causes of renal disease in CKD5-ND patients $(n=$ 53) were chronic glomerulonephritis $(n=27)$, renovascular disease or hypertension $(n=2)$, diabetic nephropathy $(n=1)$, other causes or unknown etiology $(n=23)$.

\section{PD patients}

Prevalent PD patients $(n=43)$ had been treated with biocompatible fluids: glucose-based, amino acid-based, or, for the long dwell, icodextrin-based solutions. Their median dialysis vintage time was 11.3 months. The causes of CKD were as follows: chronic glomerulonephritis $(n=14)$, renovascular disease or hypertension $(n=1)$, diabetic nephropathy $(n=6)$, other causes or unknown etiology $(n=22)$.

\section{HD patients}

Prevalent HD patients $(n=53)$ with median dialysis vintage time of 12.8 months were treated by conventional maintenance $\mathrm{HD}$ or other dialytic techniques. The causes of CKD were as follows: chronic glomerulonephritis $(n=15)$, renovascular disease or hypertension $(n=$ $5)$, diabetic nephropathy $(n=3)$, and others or unknown etiology $(n=30)$.

\section{Biochemical measurements}

Biochemical measurements of plasma high-sensitivity Creactive protein (hsCRP), albumin, creatinine, troponin, cholesterol, triglycerides, HDL-cholesterol, calcium, phosphate, alkaline phosphatase (ALP), parathyroid hormone (PTH), fibroblast growth factor 23 (FGF-23), Klotho, 25 $(\mathrm{OH})$ vitamin $\mathrm{D}$, were performed at the Clinical Chemical Laboratory of Karolinska University Hospital, Stockholm, Sweden. Human sclerostin was analyzed with ELISA kit from R\&D systems (Abingdon, UK). LDL was calculated using the Friedewald formula: [(total cholesterol) - (high- 
Table 1 Characteristics of CKD 5 patients according to tertiles of copeptin

\begin{tabular}{|c|c|c|c|c|}
\hline & Low $(n=49)$ & Middle $(n=49)$ & High $(n=51)$ & $P$ value \\
\hline \multicolumn{5}{|l|}{ General characteristics } \\
\hline Copeptin (pg/mL) & $290(216-328)$ & $380(344-438)$ & $520(455-677)$ & 0.001 \\
\hline Age (years) & $45(24-62)$ & $46(24-65)$ & $51(29-67)$ & 0.030 \\
\hline Males, n (\%) & $32(65)$ & $33(67)$ & $36(71)$ & 0.840 \\
\hline Diabetes mellitus, n (\%) & $4(8)$ & $5(10)$ & $7(13)$ & 0.663 \\
\hline Cardiovascular disease, n (\%) & $3(6)$ & $8(16)$ & $12(23)$ & 0.041 \\
\hline Previously on dialysis, n (\%) & $26(53)$ & $28(57)$ & $42(82)$ & 0.015 \\
\hline Dialysis vintage, years & $0.2(0-3.4)$ & $0.3(0-3.11)$ & $1.0(0-3.1)$ & 0.005 \\
\hline Systolic BP, (mmHg) & $133(116-156)$ & $146(125-170)$ & $144(115-180)$ & 0.008 \\
\hline Diastolic BP, (mmHg) & 80 (67-96) & $84(72-95)$ & $82(67-100)$ & 0.542 \\
\hline \multicolumn{5}{|l|}{ Nutritional status } \\
\hline Body mass index, $\left(\mathrm{kg} / \mathrm{m}^{2}\right)$ & $24.4(21-29.7)$ & $24.5(20.5-29.9)$ & $25.0(20.9-29.8)$ & 0.824 \\
\hline Lean body mass index, $\left(\mathrm{kg} / \mathrm{m}^{2}\right)^{a}$ & $18.3(15.3-20.3)$ & $18.6(14.1-22.1)$ & $18.2(14.8-21)$ & 0.888 \\
\hline Fat body mass index, $\left(\mathrm{kg} / \mathrm{m}^{2}\right)^{a}$ & $5.9(3.4-10.1)$ & $6.6(3.6-10.7)$ & $6.1(3.1-10.1)$ & 0.464 \\
\hline \multicolumn{5}{|l|}{ Markers of metabolism and nutrition } \\
\hline Hemoglobin, $(g / L)^{b}$ & $110(97-129)$ & $112(97-130)$ & $114(96-131)$ & 0.724 \\
\hline Albumin, (g/L) & $35.0(31.0-40.0)$ & $35.0(30.0-40.0)$ & $34(27.4-39.8)$ & 0.388 \\
\hline Glucose, $(\mathrm{mmol} / \mathrm{L})^{c}$ & $5.4(4.3-9.2)$ & $5.4(4.4-7)$ & $5.8(4.5-8.2)$ & 0.286 \\
\hline $\mathrm{HbA1C},(\%)^{d}$ & $33.0(19.0-40.0)$ & $33.5(25.5-40.5)$ & $33.0(23.9-40.4)$ & 0.666 \\
\hline Triglyceride, (mmol/L) & $1.2(0.6-2.3)$ & $1.3(0.6-2.3)$ & $1.4(0.7-2.6)$ & 0.072 \\
\hline Total cholesterol, (mmol/L) & $4.5(3.2-5.9)$ & $4.4(3.2-6.2)$ & $4.2(3.0-6.3)$ & 0.661 \\
\hline HDL cholesterol, (mmol/L) & $1.4(1.0-2.0)$ & $1.4(0.8-2.2)$ & $1.3(0.8-2.0)$ & 0.408 \\
\hline LDL cholesterol, (mmol/L) & $2.7(1.6-4.4)$ & $2.6(1.4-4.5)$ & $2.6(1.4-4.6)$ & 0.916 \\
\hline Plasma osmolality (mmol/kg) & $300(290-315)$ & $306(288-319)$ & $302(290-314)$ & 0.241 \\
\hline Creatinine ( $\mu \mathrm{mol} / \mathrm{L})$ & $661(484-1086)$ & $683(451-1021)$ & $829(560-1136)$ & 0.006 \\
\hline \multicolumn{5}{|l|}{ Biomarkers of inflammation } \\
\hline hsCRP, (mg/L) & $0.8(0.2-9.0)$ & $0.8(0.2-3.9)$ & $1.0(0.2-7.0)$ & 0.883 \\
\hline $\mathrm{IL}-6,(\mathrm{pg} / \mathrm{mL})^{\mathrm{e}}$ & $1.0(0.1-6.0)$ & $1.4(0.1-5.7)$ & $1.4(0-6.5)$ & 0.820 \\
\hline $\mathrm{TNF}(\mathrm{pg} / \mathrm{mL})^{f}$ & $9.0(7.2-15.2)$ & $10.8(8.3-15.2)$ & $10.5(7.6-21)$ & 0.094 \\
\hline \multicolumn{5}{|l|}{ Medications } \\
\hline$\beta$-blockers, n (\%) & $14(27)$ & $31(63)$ & $41(80)$ & $<0.0001$ \\
\hline Ca-blocker, n (\%) & $24(45)$ & $26(53)$ & $27(53)$ & 0.899 \\
\hline ACEi/ARB, n (\%) & $32(65)$ & $32(65)$ & $25(49)$ & 0.157 \\
\hline Statins, n (\%) & $15(31)$ & $16(33)$ & $21(41)$ & 0.499 \\
\hline Calcium-phosphate binders, n (\%) & $24(49)$ & $28(57)$ & $26(51)$ & 0.700 \\
\hline \multicolumn{5}{|c|}{ Biomarkers of mineral-bone disease and vascular calcification } \\
\hline Calcium, (mmol/L) & $2.3(2.1-2.6)$ & $2.3(2.0-2.5)$ & $2.3(2.0-2.5)$ & 0.814 \\
\hline Phosphate, (mmol/L) & $1.7(1.1-2.1)$ & $1.7(1.0-2.5)$ & $1.6(1.0-2.4)$ & 0.964 \\
\hline$A L P,(U / L)^{f}$ & $57(33-109)$ & $60(38-155)$ & $67(37-118)$ & 0.505 \\
\hline PTH, $(p g / m L)$ & $231(94-623)$ & $255(75-594)$ & $269(54-520)$ & 0.903 \\
\hline FGF-23, $(\mathrm{pg} / \mathrm{mL})^{\mathrm{g}}$ & $3534(223-27,943)$ & $2820(706-38,863)$ & $7486(1088-83,186)$ & 0.257 \\
\hline Klotho $(p g / m L)^{h}$ & $317(178-603)$ & 380 (197-970) & $330(119-513)$ & 0.220 \\
\hline $25(\mathrm{OH})$ vitamin D & $38(16-79.8)$ & $35(20-73)$ & $33.5(12-65.7)$ & 0.309 \\
\hline Sclerostin $(\mathrm{pg} / \mathrm{mL})^{\mathrm{i}}$ & 369 (198-939) & $409(247-682)$ & $515(271-894)$ & 0.067 \\
\hline
\end{tabular}


Table 1 Characteristics of CKD 5 patients according to tertiles of copeptin (Continued)

\begin{tabular}{lllll}
\hline & Low $(n=49)$ & Middle $(n=49)$ & High $(n=51)$ & $P$ value \\
\hline Troponin T $(\mu \mathrm{g} / \mathrm{L})$ & $17.0(5.0-53.6)$ & $19.5(3.6-71.6)$ & $32.0(1.2-68.0)$ & $\mathbf{0 . 0 3 6}$ \\
Total BMD $\left(\mathrm{g} / \mathrm{cm}^{2}\right)^{f}$ & $1.2(1.0-1.3)$ & $1.1(0.9-1.3)$ & $38(0-1946)$ & 0.100 \\
CAC score $(\mathrm{AU})^{j}$ & $0(0-700)$ & $2(0-1546)$ & & 0.055 \\
Medial calcification, n $(\%)^{\mathrm{k}}$ & & & $22(28)$ \\
$0-1$ & $34(43)$ & $23(29)$ & $25(43)$ \\
$2-3$ & $13(22)$ & $20(35)$ & $\mathbf{0 . 0 3 0}$ \\
\hline
\end{tabular}

Continuous variables are presented as median (10-90 percentile). Categorical variables are presented as number (n)/percentage (\%). Abbreviations: Systolic $B P$ Systolic blood pressure, Diastolic BP Diastolic blood pressure, $H D L$ High-density lipoprotein, $L D L$ Low-density lipoprotein, $h s C R P$ High-sensitivity C-reactive protein, IL-6 Interleukin-6, TNF Tumor necrosis factor, ACEi Angiotensin-converting enzyme, ARB Angiotensin 2 receptor blocker, ALP Alkaline phosphatase, PTH Parathyroid hormone, FGF-23 Fibroblast growth factor - 23, Total BMD Total bone mineral density, CAC score (AU) Calcification score (Agatston units) Measurements were available in following numbers of patients:

${ }^{\mathrm{a}} n=123,{ }^{\mathrm{b}} n=120,{ }^{\mathrm{c}} n=111,{ }^{\mathrm{d}} n=129,{ }^{\mathrm{e}} n=90,{ }^{\mathrm{f}} n=77,{ }^{\mathrm{g}} n=65,{ }^{\mathrm{h}} n=109,{ }^{\mathrm{i}} n=82,{ }^{\mathrm{j}} n=115,{ }^{\mathrm{k}} \mathrm{n}=137$

Every entry written in boldface in the aforementioned tables is of statistical significance $(p<0,05)$

density lipoprotein cholesterol) - (triglycerides/5)]. Tumor necrosis factor (TNF), interleukin-6 (IL-6) were analyzed by immunometric assays on an Immulite 1000 Analyzer (Siemens Healthcare Diagnostics, Los Angeles, CA, USA) using commercial kits. Plasma osmolality was measured using VAPRO Vapor Pressure Osmometer 5520 (Wescor, USA).

Blood samples for copeptin measurement were taken in patients following overnight fasting, in hemodialyzed patients samples were taken the day after HD session. Copeptin concentration was measured with a commercial enzyme immunoassay (Cloud-Clone Corp., Houston, USA) according to the manufacturer's protocol. Briefly, samples were incubated at $37^{\circ} \mathrm{C}$ with respective reagents. Optical density was read at $450 \mathrm{~nm}$ immediately. The minimum detectable dose of copeptin is typically < $6.1 \mathrm{pg} / \mathrm{mL}$. The intra-assay coefficient variation $(\mathrm{CV})$ was $<10 \%$ and the inter-assay CV was $<12 \%$.

\section{Nutritional assessments}

Body mass index (BMI) was calculated as weight in kilograms divided by the square of height in meters. Lean body mass (LBM) and fat body mass (FBM) were calculated by anthropometry based on measurements of the thickness of biceps, triceps, subscapular and supra-iliac skinfold as described previously [20], using equations by Siri [21]. LBM index and FBM index were calculated according to Kyle et al. [22]. Bone mineral density (BMD) was determined by dual-energy X-ray absorptiometry (DXA).

\section{CAC score}

Cardiac CT scans were performed using a 64-channel detector scanner (Lightspeed VCT; General Electric (GE) Healthcare, Milwaukee, WI). CAC scores were expressed in Agatston units, the protocol and measurements as described previously in detail [13]. Total CAC score was calculated as the sum of CAC scores in the left main artery, the left anterior descending artery, the left circumflex artery, and the right coronary artery.

\section{Vascular scoring by histology}

Inferior epigastric arteries were collected from patients within $20 \mathrm{~min}$ from the start of kidney transplantation procedure. After preparation, the sections were then stained with hematoxylin and eosin and von Kossa staining before evaluation by one experienced pathologist. The degree of medial calcification was quantified semiautomatically according to method described previously [13] and graded 0 to 3 , where 0 indicated no calcification and 3 the highest degree of calcification. Patients graded as 0 and 1 represented no-minimal vascular calcification, and those graded 2 and 3 represented moderateextensive vascular calcification.

\section{Statistical analyses}

Continuous data are expressed as median (10th to 90th percentile) and nominal data as percentage. $P$ value was set at $p<0.05$. For comparisons between three groups non-parametric Kruskal-Wallis ANOVA-test was used for continuous variables and Chi-square test was used for nominal variables. Associations between variables were determined using non-parametric Spearman rank correlation analysis. Multivariate linear regression analyses of copeptin were used and results were shown as standardized $\beta$ regression coefficients. We performed multinomial logistic regression analysis to examine factors associated for determinants of vascular calcification 0 and 1 score versus 2 and 3. Statistical analyses were performed using statistical software SAS version 9.4 (SAS Campus Drive, Cary, NC, USA) and Stata 16 (Stata Corporation, College Station, TX, USA).

\section{Results}

Demographics and clinical characteristics of the examined patients according to copeptin tertiles are shown in Table 1. Patients with higher copeptin were older, had 
higher systolic blood pressure and were more frequently diagnosed with CVD. In addition, higher percentage of these patients had been undergoing dialysis with longer vintage. Characteristics of the studied CKD5 patients according to dialysis dependence are presented in Additional file 1: Table S1. There was no difference in copeptin concentration between PD and HD patients (427 vs. $412 \mathrm{pg} / \mathrm{mL}, p=1.0$ ), while plasma copeptin was significantly lower in CKD5-ND patients compared to PD and HD patients (351 vs. $427 \mathrm{pg} / \mathrm{mL}, p=0.02$; and 351 vs. $412 \mathrm{pg} / \mathrm{mL}, \quad p=0.001$, respectively). Plasma osmolality and copeptin correlated in CKD5-ND patients (Rho $=0.28 ; p=0.04$ ), whereas in the two groups of dialyzed patients this association was not observed. In Spearman's rank correlations (Rho), copeptin concentrations were significantly associated with age $(\mathrm{Rho}=0.21$; $p=0.011$ ), presence of CVD ( $\mathrm{Rho}=0.20 ; p=0.013)$, systolic blood pressure (Rho $=0.20 ; p=0.01$ ), triglycerides (Rho $=0.22 ; p=0.007)$, serum creatinine ( $\mathrm{Rho}=0.26 ; p=$ $0.001)$, TNF (Rho $=0.27 ; p=0.016)$, sclerostin $(\mathrm{Rho}=0.22$; $p=0.049$ ), troponin $\mathrm{T}$ ( $\mathrm{Rho}=0.27 ; p=0.001$ ), CAC score (Rho $=0.27 ; p=0.003)$ and histological scoring of medial calcification (Rho $=0.21 ; p=0.016)$. Copeptin did not have significant association with gender and diabetes mellitus.

When CAC scores were categorized as $\mathrm{CAC}=0, \mathrm{CAC} \geq$ 100 and $C A C \geq 1000$, we found no statistically significant differences in copeptin concentration between the group of $C A C=0$ and $C A C \geq 100$ (372.9 [range 259.4-529.8] vs 426.8 [range 242.3-651.8], $p=0.76$ ) but there was statistically significant difference between the group of $\mathrm{CAC}=0$ and $C A C \geq 1000$ (372.9 [range 259.4-529.8] vs 453.9 [range 333.4-681.1], $\mathrm{p}=0.001$ ).

A multivariate linear regression model for determinants for plasma copeptin showed that 1-SD increase of age, 1-SD increase of serum creatinine and 1-SD increase of triglycerides were associated with higher plasma copeptin levels (Table 2). Patients with histological signs of moderate-extensive $\mathrm{VC}$ had significantly higher copeptin levels (Fig. 1).

Table 2 Multivariate linear regression for the prediction of copeptin, pg/ml ( $n=149)$, expressed as standardized $\beta$ coefficients with other variables

\begin{tabular}{ll}
\hline & Copeptin (adj $\left.\mathrm{r}^{2}=0.14\right)$ \\
\hline Parameters & standardized $\beta$ ( $\mathrm{p}$ value) \\
1-SD increase of age, years & $\mathbf{0 . 2 5}(\mathbf{0 . 0 0 2})$ \\
Gender (male, female) & $-0.08(0.31)$ \\
1-SD increase of serum creatinine $(\mu \mathrm{mol} / \mathrm{l})$ & $\mathbf{0 . 2 6 ( \mathbf { 0 . 0 0 3 } )}$ \\
1-SD increase of triglyceride $(\mathrm{mmol} / \mathrm{L})$ & $\mathbf{0 . 1 6 ( \mathbf { 0 . 0 3 } )}$ \\
Systolic blood pressure $(\mathrm{mmHg})$ & $0.14(0.07)$ \\
\hline
\end{tabular}

Every entry written in boldface in the aforementioned tables is of statistical significance $(p<0,05)$

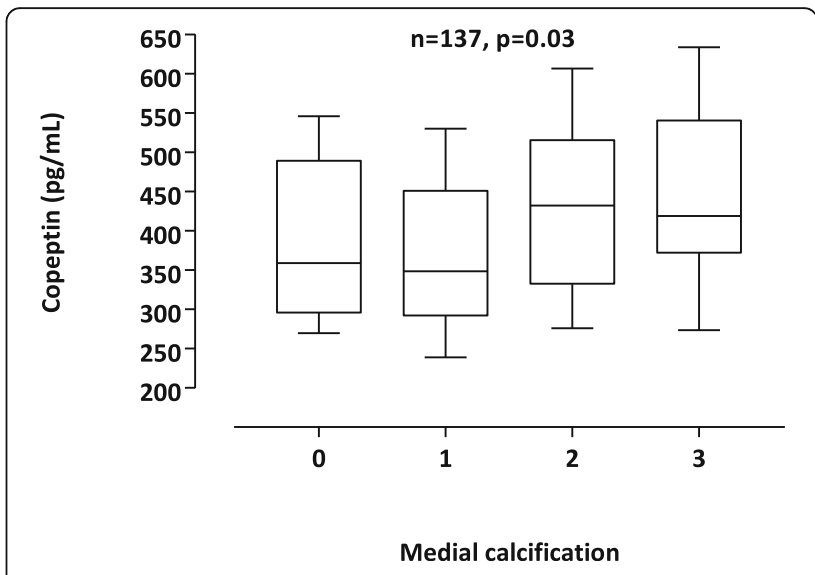

Fig. 1 Plasma copeptin levels in relation to medial calcification

Univariate analysis showed no significant correlations between biomarkers of mineral-bone disease and $\mathrm{VC}$; however, a positive correlation between hsCRP and VC was found (Rho $=0.18, p=0.03$ ). Receiver operating characteristic (ROC) curves for prediction of $\mathrm{VC}$ showed that the area under the curve (AUC) was for copeptin - AUC 0.64 $(p=0.02)$ and for hsCRP - AUC 0.54 ( $\mathrm{p}=0.02)$. Multivariate logistic regression analysis showed that $1-\mathrm{SD}$ increase of age, male gender, presence of diabetes and 1-SD increase of copeptin were significantly associated with the presence of moderate-extensive VC (Table 3).

\section{Discussion}

We report that in CKD5 patients, high plasma copeptin levels are associated with age, CVD, dialysis vintage, systolic blood pressure and serum creatinine. The chief finding of the study was that elevated copeptin levels were associated with biopsy-verified media calcification. Multivariate analysis showed that copeptin was related to extent of $\mathrm{VC}$ independently of age, male gender and the presence of diabetes.

Vascular calcification, viewed in the past as a passive process, is now recognized as an active phenomenon [23].

Table 3 Multivariate logistic regression model for determinants of VC 0 and 1 score versus 2 and $3(n=137)$

\begin{tabular}{|c|c|}
\hline & $V C$ (pseudo $\left.r^{2}=0.29\right)$ \\
\hline Parameters & Odds Ratio 95\%Cl ( $p$ value) \\
\hline 1-SD increase of age, years & $2.5(1.5-4.1)(0.001)$ \\
\hline Gender (male, female) & $4.4(1.6-11.1)(0.003)$ \\
\hline Diabetes (No, Yes) & $23.2(2.5-210.5)(0.005)$ \\
\hline CKD5-ND vs HD & $0.7(0.4-1.8)(0.42)$ \\
\hline CKD5-ND vs PD & $0.5(0.4-1.8)(0.42)$ \\
\hline 1-SD increase of hsCRP (mg/L) & $0.7(0.4-1.3)(0.254)$ \\
\hline 1-SD increase of Copeptin, (pg/ml) & $1.6(1.1-2.6)(0.043)$ \\
\hline
\end{tabular}

Every entry written in boldface in the aforementioned tables is of statistical significance $(p<0,05)$ 
The mechanisms underlying increased VC in CKD are not fully explained, but they involve disorders in calcium and phosphorus metabolism, and disturbed homeostasis in pro- and anti-calcification factors. Recently, novel tests, such as T50 functional test, have been developed to help in the determination of calcification propensity in CKD5 patients [24]. It has also been shown that uremia induces the differentiation of vascular smooth muscle cells (VSMCs) - the most important component of the media of arteries - into osteoblast-like cells due to up-regulation of transcription factors, such as Runt-related transcription factor 2 (Runx2) and Msh homebox 2 (Msx2) and increased alkaline phosphatase activity [25]. These changes contribute to the calcification of VSMC, in a way similar to bone formation (i.e. ossification).

To the best of our knowledge, this is the first study to show a link between plasma copeptin level and the degree of $\mathrm{VC}$ in $\mathrm{CKD}$. Emerging research suggest that vasopressin plays important role in the progression of
CKD and hypertension via stimulation of the renin angiotensin aldosterone system (RAAS) leading to vasoconstriction and higher systemic and glomerular blood pressure [18]. Direct effects on VSMCs also result in vasoconstriction [26]. By modulating both the number and size of VSMC, AVP may play a direct role in the development of chronic vascular disease. It has been reported that AVP activates intracellular $\mathrm{Ca}^{2+}$ and protein kinase $C(\mathrm{PC})$ via the activation of phospholipase (PL) C and $\mathrm{D}$ leading to augmentation of phosphate transport during proliferation of VSMC [27, 28]. Among other actions, AVP also stimulates secretion of endothelin-1 (ET-1) from endothelial cells which enhances endothelial dysfunction and coagulation disorders as well as increases cellular sodium-dependent phosphate transport via Pit1 and Pit2 [29, 30]. This suggests, that in the uremic hyperphosphatemic milieu, there is intensed entry of phosphate into VSMC. Furthermore, an increase in cellular phosphate can increase cellular calcification



Fig. 2 Mechanisms of vascular calcification in CKD. In the setting of uraemic milieu, activation of renin-angiotensin and vasopressin systems, upregulation of sodium-dependent phosphate transporter Pit-1 promotes osteochondrocytic transformation and apoptosis of vascular smooth muscle cell (VSMC) and, in consequence, accelerated vascular calcification. Dashed lines show possible copeptin links with the process 
by different mechanisms: an increase in calcium $\mathrm{x}$ phosphate product, change of VSMC to a bone-producing cell phenotype and cell apoptosis that associates with increased secretion of pro-calcific factors [31]. A schematic presentation of mechanisms leading to $\mathrm{VC}$ including potential links with copeptin is shown in Fig. 2.

Our study showed positive correlation between plasma osmolality and copeptin in the group of nondialyzed CKD5 patients, whereas such relationship was not observed in PD and HD groups, and nondialyzed patients had also lower plasma copeptin levels than the other two groups. This accords with Ettema et al. [32] who examined copeptin levels in patients with different stages of CKD, including CKD5. They observed positive correlation between plasma osmolality and copeptin in patients with CKD and higher residual renal function (RRF) associated with lower plasma copeptin levels in HD patients which underscore the importance of RRF in the clearance of copeptin. Finally, they observed that both vasopressin and copeptin were removed during HD (with higher dialyzer clearance rate of vasopressin). We recently [33] reported that in patients undergoing $\mathrm{PD}$, dialysate copeptin was positively correlated with plasma copeptin, indicating that also peritoneal clearance of copeptin may contribute to explain why there was no association between plasma copeptin and osmolality among the dialyzed patients in the current study.

A noteworthy finding of the study is the strong correlation of plasma copeptin with the use of $\beta$ blockers. To the best of our knowledge, such relationship has not been described before. Since the mechanism (s) of this correlation remain unclear, further studies are needed to clarify if this is merely a reflection of confounding by indication or if $\beta$-blockers do affect copeptin.peter.

The results of the present study should be considered given some important limitations. First, we examined CKD5 patients with different types of treatment; i.e. conservative, HD and PD. Moreover, since the group in which vascular biopsies were available was selected for LD-Rtx and thus represent a selected group of "healthier" CKD5 patients. Thus, since diabetic nephropathy was underrepresented compared to the typical CKD5 population the association between $\mathrm{VC}$ and copeptin may have been underestimated. Third, as in any observational study, cause-effect relations cannot be determined. Finally, although the circulating concentration of copeptin directly reflects that of AVP and copeptin shows the same response to osmotic changes as AVP in CKD, copeptin is reported to increase more than vasopressin suggesting that the clearance rate of copeptin is more markedly reduced than for vasopressin when GFR decreases. Thus, while copeptin reflects vasopressin levels, in CKD stages 4 and 5, a correction for renal function might be required [14, 32].

\section{Conclusions}

High circulating levels of copeptin in CKD5 were found to associate with total CAC score (by cardiac $\mathrm{CT}$ ), and with the degree of medial vascular calcification (by histology of arterial biopsies) even after adjusting for confounders. Further studies are needed to resolve if copeptin is simply a marker or a player in the complex uremic vascular calcification process.

\section{Supplementary information}

Supplementary information accompanies this paper at https://doi.org/10. 1186/s12882-020-1710-6.

Additional file 1: Table S1. Characteristics of CKD 5 patients stratified according to dialysis-dependence.

\section{Abbreviations}

AVP: Arginine vasopressin; CAC: Coronary artery calcification; CKD: Chronic kidney disease; CVD: Cardiovascular disease; ET-1: Endothelin 1; HD: Hemodialysis; LD-Rtx: Living donor renal transplantation; PD: Peritoneal dialysis; VC: Vascular calcification; VSMC: Vascrular smooth muscle cell

\section{Acknowledgements}

We thank all patients who participated in the study and those who carried out the extensive clinical and laboratory work in the clinical investigational unit and the renal laboratory at Dept. of Renal Medicine, Karolinska University Hospital specially Ann-Christin Bragfors Helin.

\section{Authors' contributions}

Research idea and study design: EG, RJ. Data aquisition: $A R Q, O H, P B, P S$. Data analysis: EG, ARQ, LD, BL, PS. Statistical analysis: ARQ. Drafting the article: EG. Supervision of manuscript: PS. Revision process: ARQ, LD, BL, OH, MS, TB, $J R, P B, R J, P S$. Specific contribution to scoring of arterial biopsies: MS. Specific contribution to analysis of $C T$ heart radiographies: TB, JR. All authors have read and approved the final version of the manuscript.

\section{Funding}

This study was supported by a grant from Baxter Healthcare to Baxter Novum, Department of Clinical Science, Intervention and Technology, Karolinska Institutet. The study also benefited from generous support from Karolinska Institutet Diabetes Theme Center (PS), Heart and Lung Foundation (PS), Njurfonden (PS), and Westmans Foundation (PS). This study also received funding from the European Union's Horizon2020 research and innovation program under the Marie Sklodowska-Curie grant agreement No 722609. The funders of this study had no role in collection, data analysis and/or interpretation of data in this study.

\section{Availability of data and materials}

The datasets used and and analysed during the current study are available from the corresponding author on reasonable request.

\section{Ethics approval and consent to participate}

The study was performed in accordance with the principles of the Declaration of Helsinki and was approved by the Ethics Committee of the Karolinska Institutet (EPN). Written informed consent was obtained from each patient. 


\section{Consent for publication}

Not applicable

\section{Competing interests}

Baxter Novum is the result of a grant from Baxter Healthcare to Karolinska Institutet. Bengt Lindholm is employed by Baxter Healthcare Corporation. The other authors declare no conflict of interest.

\section{Author details}

'Division of Renal Medicine and Baxter Novum, Department of Clinical Science, Intervention and Technology, Karolinska Institutet, Campus Flemingsberg, Stockholm, Sweden. ${ }^{2}$ Department of Nephrology, Transplantology and Internal Medicine, Pomeranian Medical University, Al. Powstancow Wlkp. 72, 70-111 Szczecin, Poland. ${ }^{3}$ Cardiovascular, Renal and Metabolism Safety, Clinical Pharmacology \& Safety Sciences R\&D, AstraZeneca, Gothenburg, Sweden. ${ }^{4}$ Division of Medical Imaging and Technology, Department of Clinical Science, Intervention and Technology, Karolinska Institutet, Campus Flemingsberg, Stockholm, Sweden. ${ }^{5}$ Division of Renal Diseases and Hypertension, School of Medicine, University of Colorado, Denver, CO, USA.

\section{Received: 17 August 2019 Accepted: 30 January 2020}

\section{Published online: 07 February 2020}

\section{References}

1. Quinn MP, Cardwell CR, Kee F, Maxwell AP, Savage G, McCarron P, et al. The finding of reduced estimated glomerular filtration rate is associated with increased mortality in a large UK population. Nephrol Dial Transplant. 2011; 26(3):875-80.

2. Dai L, Golembiewska E, Lindholm B, Stenvinkel P. End-stage renal disease, Inflammation and Cardiovascular Outcomes. Contrib Nephrol. 2017;191:32-43

3. Stenvinkel P. Chronic kidney disease: a public health priority and harbinger of premature cardiovascular disease. J Intern Med. 2010;268(5):456-67.

4. Stenvinkel P, Pecoits-Filho R, Lindholm B. Coronary artery disease in endstage renal disease: no longer a simple plumbing problem. J Am Soc Nephrol. 2003;14(7):1927-39.

5. Sigrist M, Bungay P, Taal MW, Mclntyre CW. Vascular calcification and cardiovascular function in chronic kidney disease. Nephrol Dial Transplant. 2006;21(3):707-14

6. Civilibal M, Caliskan S, Adaletli I, Oflaz H, Sever L, Candan C, et al. Coronary artery calcifications in children with end-stage renal disease. Pediatr Nephrol. 2006;21(10):1426-33

7. London GM, Guérin AP, Verbeke FH, Pannier B, Boutouyrie P, Marchais SJ, et al. Mineral metabolism and arterial functions in end-stage renal disease: potential role of 25-hydroxyvitamin D deficiency. J Am Soc Nephrol. 2007; 18(2):613-20.

8. Han D, Lee JH, Hartaigh B, Min JK. Role of computed tomography screening for detection of coronary artery disease. Clin Imaging. 2016;40(2):307-10.

9. Takayama Y, Yasuda Y, Suzuki S, Shibata Y, Tatami Y, Shibata K, et al. Relationship between abdominal aortic and coronary artery calcification as detected by computed tomography in chronic kidney disease patients. Heart Vessel. 2016;31(7):1030-7.

10. Ketteler M, Block GA, Evenepoel P, et al. Executive summary of the 2017 KDIGO Chronic Kidney Disease-Mineral and Bone Disorder (CKD-MBD) Guideline Update: what's changed and why it matters. Kidney Int. 2017;92: 26-36 Kidney Int. 2017;92(6):1558

11. Alderson HV, Ritchie JP, Green D, Chiu D, Kalra PA. Potential for biomarkers of chronic kidney disease-mineral bone disorder to improve patient care. Nephron Clin Pract. 2013;124(3-4):141-50.

12. Nascimento MM, Hayashi SY, Riella MC, Lindholm B. Elevated levels of plasma osteoprotegerin are associated with all-cause mortality risk and atherosclerosis in patients with stages 3 to 5 chronic kidney disease. Braz J Med Biol Res. 2014:47(11):995-1002.

13. Qureshi AR, Olauson H, Witasp A, Haarhaus M, Brandenburg V, Wernerson A et al. Increased circulating sclerostin levels in end-stage renal disease predict biopsy-verified vascular medial calcification and coronary artery calcification. Kidney Int. 2015:88(6):1356-64.

14. Golembiewska E, Machowska A, Stenvinkel P, Lindholm B. Prognostic value of Copeptin in chronic kidney disease: from general population to endstage renal disease. Curr Protein Pept Sci. 2017;18(12):1232-43.
15. Christ-Crain M, Fenske W. Copeptin in the diagnosis of vasopressindependent disorders of fluid homeostasis. Nat Rev Endocrinol. 2016:12(3): 168-76.

16. Velho G, Bouby N, Hadjadj S, Matallah N, Mohammedi K, Fumeron F, et al. Plasma Copeptin and renal outcomes in patients with type 2 diabetes and albuminuria. Diabetes Care. 2013;36(11):3639.

17. Ponte B, Pruijm M, Ackermann D, Vuistiner P, Guessous I, Ehret G, et al. Copeptin is associated with kidney length, renal function, and prevalence of simple cysts in a population-based study. J Am Soc Nephrol. 2015;26(6): 1415-25.

18. Afsar B. Pathophysiology of copeptin in kidney disease and hypertension. Clin Hypertens. 2017;23:13

19. Bjornstad P, Maahs DM, Jensen T, Lanaspa MA, Johnson RJ, Rewers M, et al. Elevated copeptin is associated with atherosclerosis and diabetic kidney disease in adults with type 1 diabetes. J Diabetes Complicat. 2016:30(6): 1093-6.

20. Durnin JV, Womersley J. Body fat assessed from total body density and its estimation from skinfold thickness: measurements on 481 men and women aged from 16 to 72 years. Br J Nutr. 1974;32(1):77-97.

21. Siri WE. Body composition from fluid spaces and density: analysis of methods. 1961. Nutrition. 1993;9(5):480-91 discussion , 92.

22. Kyle UG, Schutz Y, Dupertuis YM, Pichard C. Body composition interpretation. Contributions of the fat-free mass index and the body fat mass index. Nutrition. 2003:19(7-8):597-604.

23. Nitta K. Vascular calcification in patients with chronic kidney disease. Ther Apher Dial. 2011:15(6):513-21.

24. Pasch A, Block GA, Bachtler M, Smith ER, Jahnen-Dechent W, Arampatzis S, et al. Blood calcification propensity, cardiovascular events, and survival in patients receiving hemodialysis in the EVOLVE trial. Clin J Am Soc Nephrol. 2017;12(2):315-22.

25. Shroff RC, McNair R, Figg N, Skepper JN, Schurgers L, Gupta A, et al. Dialysis accelerates medial vascular calcification in part by triggering smooth muscle cell apoptosis. Circulation. 2008;118(17):1748-57.

26. Campbell-Boswell M, Robertson AL. Effects of angiotensin II and vasopressin on human smooth muscle cells in vitro. Exp Mol Pathol. 1981;35(2):265-76.

27. Nishiwaki-Yasuda K, Suzuki A, Kakita A, Sekiguchi S, Asano S, Nishii K, et al. Vasopressin stimulates Na-dependent phosphate transport and calcification in rat aortic smooth muscle cells. Endocr J. 2007;54(1):103-12.

28. Nemenoff RA. Vasopressin signaling pathways in vascular smooth muscle. Front Biosci. 1998;3:d194-207.

29. Spatz M, Stanimirovic D, Bacic F, Uematsu S, McCarron RM. Vasoconstrictive peptides induce endothelin-1 and prostanoids in human cerebromicrovascular endothelium. Am J Phys. 1994;266(3 Pt 1):C654-60.

30. Masukawa H, Miura Y, Sato I, Oiso Y, Suzuki A. Stimulatory effect of endothelin-1 on Na-dependent phosphate transport and its signaling mechanism in osteoblast-like cells. J Cell Biochem. 2001;83(1):47-55.

31. Rabkin SW. Endothelin but not angiotensin II may mediate hypertensioninduced coronary vascular calcification in chronic kidney disease. Int Nephrol. 2011;2011:516237.

32. Ettema EM, Heida J, Casteleijn NF, Boesten L, Westerhuis R, Gaillard CAJM, et al. The effect of renal function and hemodialysis treatment on plasma vasopressin and Copeptin levels. Kidney Int Rep. 2017;2(3):410-9.

33. Fijałkowski M, Safranow K, Lindholm B, Ciechanowski K, Muraszko AM, Dołęgowska $B$, et al. Dialysate copeptin and peritoneal transport in incident peritoneal dialysis patients. Int Urol Nephrol. 2019:51(9):1667-73.

\section{Publisher's Note}

Springer Nature remains neutral with regard to jurisdictional claims in published maps and institutional affiliations. 